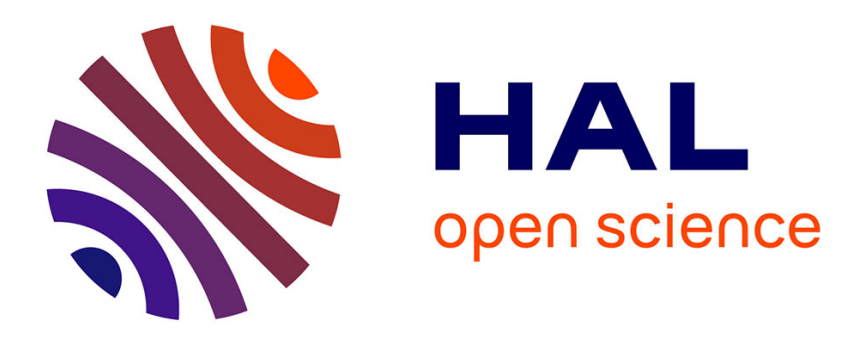

\title{
Les grands traits de l'histoire forestière
}

Marcel Lortie

\section{To cite this version:}

Marcel Lortie. Les grands traits de l'histoire forestière. Revue forestière française, 1979, 31 (S), pp.22-27. 10.4267/2042/21333 . hal-03397232

\section{HAL Id: hal-03397232 \\ https://hal.science/hal-03397232}

Submitted on 22 Oct 2021

HAL is a multi-disciplinary open access archive for the deposit and dissemination of scientific research documents, whether they are published or not. The documents may come from teaching and research institutions in France or abroad, or from public or private research centers.
L'archive ouverte pluridisciplinaire HAL, est destinée au dépôt et à la diffusion de documents scientifiques de niveau recherche, publiés ou non, émanant des établissements d'enseignement et de recherche français ou étrangers, des laboratoires publics ou privés. 


\section{LES GRANDS TRAITS DE L'HISTOIRE FORESTIËRE}

Marcel LORTIE

Retracer l'histoire forestière du Québec, c'est le plus souvent raconter l'histoire de ce pays, car le Québec est d'abord un territoire forestier. Les quelques zones urbaines et rurales qu'on y trouve ont été arrachées à la forèt qui, quelques siècles plus tard, est toujours prête à reprendre ses droits sur les territoires qu'elle a perdus.

II nous a semblé que le cadre du numéro spécial de la Revue forestière française sur le Québec forestier se prêtait davantage à un survol plutōt qu'à une étude en profondeur. Aussi, le lecteur trouvera-t-il une description sommaire des attitudes manifestées par les habitants de la Nouvelle-France, du BasCanada et enfin du Québec et des tendances dans I'utilisation de la matiére ligneuse depuis la découverte du pays par Jacques Cartier en 1534 et la fondation de Québec par Samuel de Champlain en 1608.

Au début de la Nouvelle-France, les habitants ont combattu la forêt. Les premiers à en percevoir l'importance éventuelle sont les rois de France et d'Angleterre. Le développement de l'exploitation forestière au début du XIX' siè. cle est largement attribuable au blocus continental qui força l'Angleterre à se rabattre sur ses colonies pour satisfaire aux besoins de sa marine royale. Augmentation démographique, développement des machines, amélioration des modes de transport sont autant d'événements qui ont accéléré la production en bois de sciage. Au début du $X X^{e}$ siècle, c'est l'avènement de l'industrie des pâtes et papiers qui occupe maintenant la première place de l'activité forestière au Québec. Ce sont ces étapes que nous avons tenté de présenter brièvement.

Si Cartier et Champlain sont émerveillés de l'épaisseur et de la densité de la forêt, les premiers colons la jugent plutôt comme une ennemie à éliminer. Sa destruction par le feu permet d'éloigner l'Iroquois. De 1627 jusqu'à sa dissolution, la Compagnie des CentAssociès, formée pour assurer la colonisation du pays, ne s'intéresse pas au bois, bien qu'elle ait obtenu le monopole de son com. merce comme celui aussi de la traite des fourrures.

En 1663, la Compagnie remet au roi de France, propriété eł gestion de la colonie. A la place, le monarque établit le Conseil souverain de Québec et institue la fonction d'intendant destinée à prendre en main l'administration du pays. Dans ses instructions à Jean Talon, premier intendant à Québec en 1665 , le roi lui recommande "de veiller à la conservation des bois utiles"

C'est précisément vers cette date qu'on commence à imaginer les possibilités des bois de la colonie. Pierre Boucher (1664) note que le pin et l'épinette seraient des bois propres à faire des mâts de navires. Vers 1670, Talon assure le roi que ce pays prendra la reléve lorsque "les forêts de l'ancienne France seront altérées". 


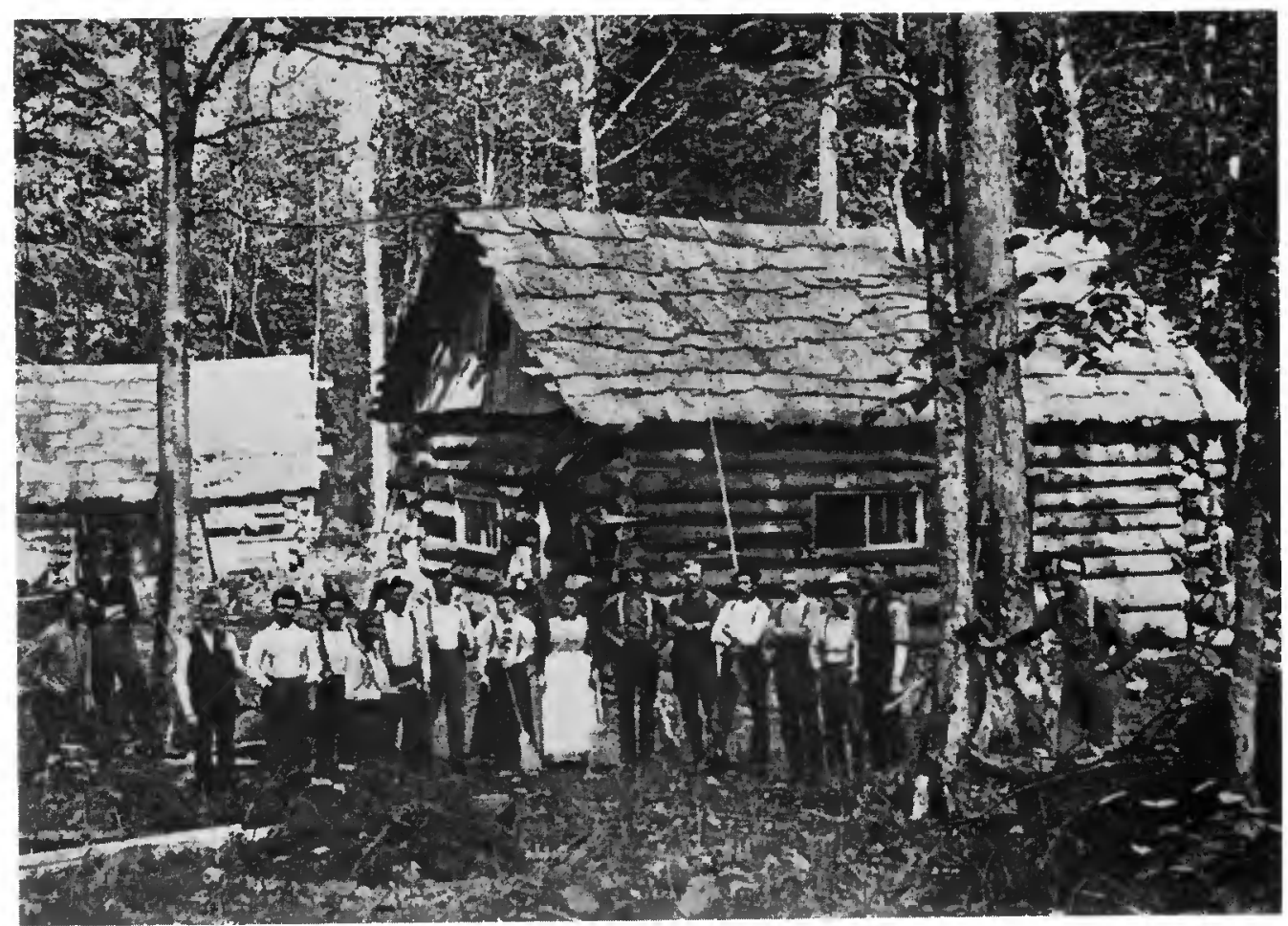

Photos Office du film du Québec

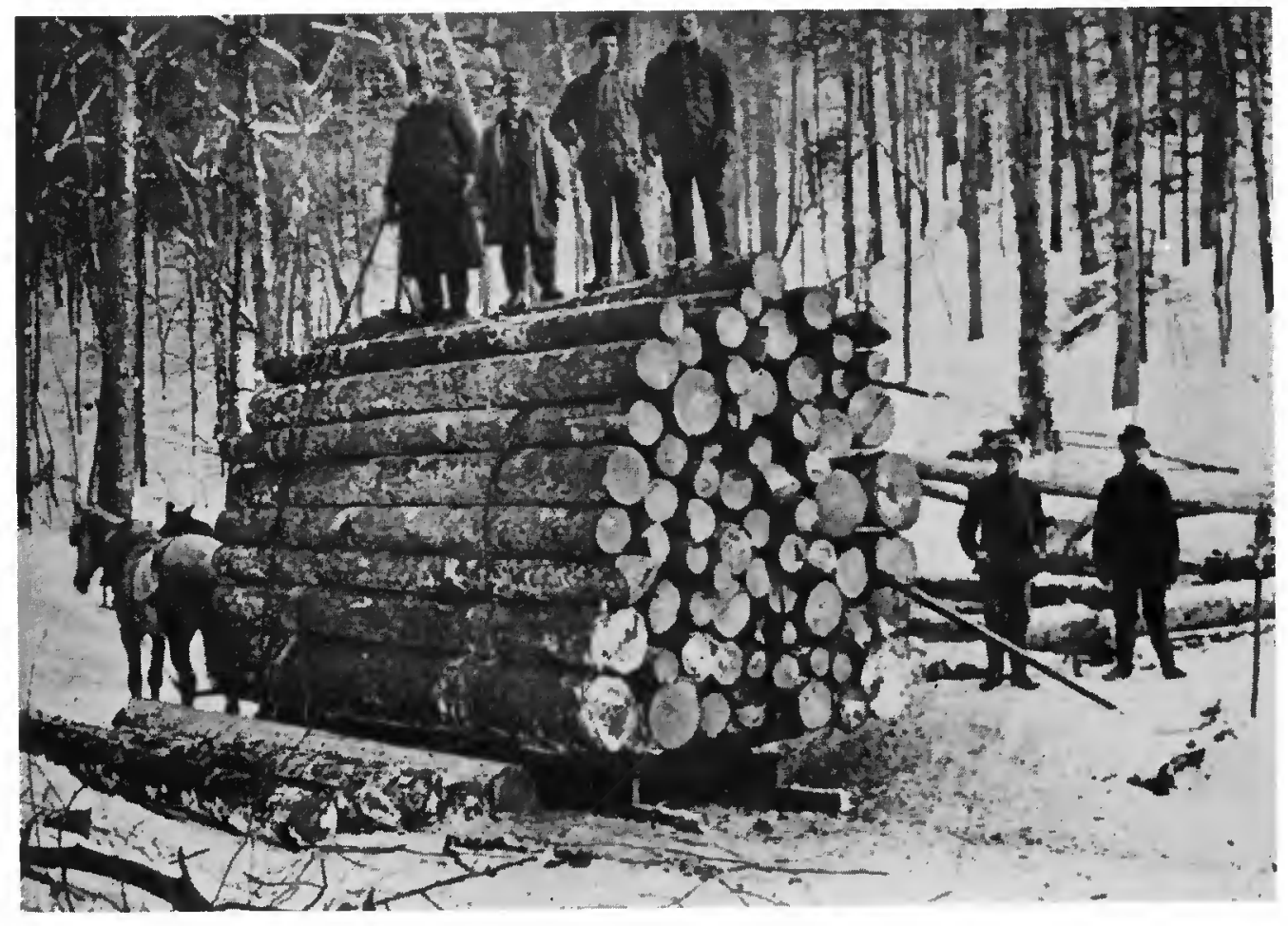




\section{LORTIE}

A partir de 1672 , toute concession de terre est sujette à une réserve de tous les bois de chêne en faveur de la marine royale. Le pin sera l'objet d'une même réserve quelques annèes plus tard. Vers le même temps, bien qu'en volume limité et au prix de nombreuses difficultés, des exportations de bois s'organisent vers la métropole.

Cependant, la fin du régime français n'aura pas vu la réalisation de la prédiction de l'intendant Dupuy qui, au début du XVIII siècle, affirmait que le bois deviendrait "le fruit du Canada qui va succéder à la pelleterie ". Même si la forêt n'est plus infestée d'ennemis, son brûlage assure le défrichement rapide des concessions. Tout au plus, prend-on soin d'abattre au préalable les arbres nécessaires à satisfaire les besoins locaux essentiels comme l'abri et le chauffage. Les seuls maigres efforts pour développer l'industrie forestière durant cette période portent sur la construction de navires et la fabrication de mâtures et de bois de marine. Les exploitations se limitent donc au territoire bordant le Saint-Laurent ou dans les forêts du bassin inférieur de quelques affluents.

Les premières préoccupations de la monarchie anglaise rejoignent celles du roi de France. A l'aube du gouvernement civil créé en 1774, les instructions royales au gouverneur Carleton exigent la mise en réserve des peuplements de pins pour la marine royale.

Cependant, dans la colonie, des changements démographiques s'amorcent. Le gouvernement de Londres doit satisfaire officiers et soldats anglais désireux de s'établir au Canada. Puis, ce sont les Loyalistes qui fuient la révolution américaine. De nombreuses concessions sont alors défrichées au sud du pays.

Au lendemain de l'Acte de 1791, alors que le nouveau parlement du Bas-Canada a d'autres préoccupations, gouverneurs et fonctionnaires anglais s'octroient et concèdent d'importantes superficies forestières. Durant la seule période de 1796 à 1809 , ce mode de concessions gratuites et en tenure libre permet à quelque 70 individus de s'accaparer plus de 660000 hectares des meilleures terres. S'il est impossible de determiner la proportion de ces terres encore sous régime forestier aujourd'hui, ce mode de concession a contribué à augmenter le régime de propriété forestière privée.

Au début du XIX siècle, l'exploitation et le commerce du bois commencent à prendre de l'importance. Le blocus continental oblige Londres à satisfaire ses besoins en bois à partir des colonies. En 1806, le premier d'une multitude de radeaux de pin équarri descend la rivière Gatineau à destination du port de Québec. Ce manège devait se poursuivre pendant une centaine d'années. Peu à peu cependant, l'énorme proportion de déchets laissés en forêt à la suite de cette exploitation ainsi que la diminution des arbres à fort diamètre, obligent les industriels à établir des scieries. Déjà en 1815 , le bois débité en planches constitue un volume important d'exportation en matériel de plus en plus recherché en Angleterre.

Si l'octroi de permis de coupe par le gouvernement anglais commence avec le début du siècle, ce n'est qu'en 1820 que le gouvernement du Bas-Canada commence à percevoir des droits de coupes sur les bois coupés sur les terres publiques. Puis en 1827, les autorités commencent à affermer les forêts en vendant des permis de coupe de bois sur les terres publiques. Après avoir subi diverses modifications aux règlements initiaux, ce système d'affermage fait l'objet d'une législation en 1849. La portée générale de cette loi devait rester inchangée pendant plus de cent ans.

Le commerce du bois du Bas-Canada puis du Québec après la Confédération, subit diverses vicissitudes. Vers 1825 , les exportations des bois canadiens commencent vers les ÉtatsUnis. Après diverses vicissitudes entre 1860 et 1865, ce commerce avec les Américains se révèle plus important que le marché traditionnel de la Grande-Bretagne. Ces deux pays constitueront longtemps les principaux marchés pour l'industrie du sciage du Québec. Son importance cependant déclinera après 1860.

Lors de la Confédération, en 1867, l'administration du domaine boise est confièe aux provinces. Au Québec, cette administration relève d'un commissaire puis quelques années plus 


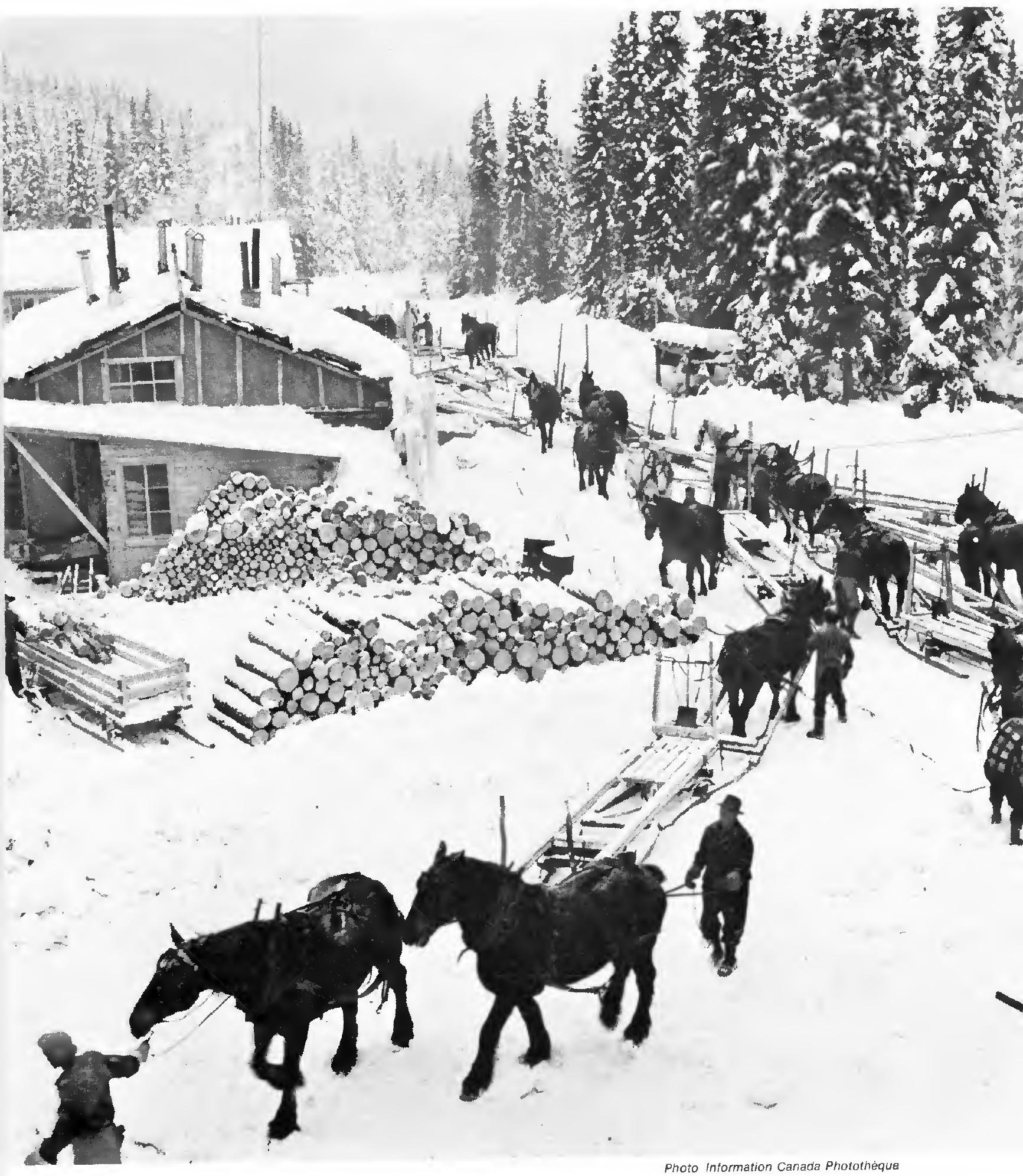




\section{LORTIE}

tard du ministère des Terres et Forêts jusqu'en 1979 où l'administration forestiére sera incorporée dans un ministère de l'Énergie et des Ressources.

Le rythme de l'exploitation forestière se pour. suit comme avant la Confédération. De plus en plus, cependant, les scieries se développent; en 1875 , leur nombre dépasse la centaine et elles sont réparties partout dans le Québec habité, autant pour satisfaire les besoins locaux que l'exportation.

A partir des années 1920, l'industrie du sciage subit les contrecoups d'une industrie montante, celle des pâtes et papiers, et de la mise en marché des bois de forte dimension de la Colombie britannique, favorisée par des moyens de transport améliorés. Plusieurs grandes scieries disparaissent, de petits établissements faciles à déménager viennent s'installer pour utiliser les restes de la première récolte ou pour subvenir aux besoins locaux.

Après l'utilisation des arbres de qualité par l'industrie du sciage, l'industrie des pâtes et papiers exigea surtout une grande quantité de matière ligneuse à bon marché. Les compagnies détentrices d'immenses forêts affermées se lancent alors dans la construction d'usines de pâtes et papiers. Actuellement au Québec, les produits de la pâte et du papier tiennent le premier rang de l'industrie forestière et la province détient toujours le premier rang dans la production canadienne.

Depuis les années 1960, on assiste à des tendances nouvelles dans l'économie forestière au Québec. Des mesures propres à assurer une meilleure utilisation des forêts se multiplient. L'industrie des pâtes et papiers est invitée à utiliser les copeaux, produit accessoire de l'industrie du sciage. Celle-ci, à son tour, reçoit un appui gouvernemental non déguisé en raison de l'importance de la valeur ajoutée de sa production. Le déroulage des billes prend sa part du marché et on voit maintenant un intérêt grandissant pour la fabrication de panneaux de particules ou d'agglomérés.

En même temps, on assiste au développement d'une foresterie oú la production de matière ligneuse se voit confrontée à d'autres objectifs comme la conservation de la faune, la récréation, la protection intégrale de parcelles, les réserves écologiques, l'esthétique, etc. Qui sait ce que sera la contribution de la forêt québécoise en terme de biomasse à la solution des problèmes de plus en plus aigus en énergie?

En réponse à ces nouveaux besoins, le Québec a entrepris des changements profonds à sa législation âgée. On a amorcé la rétrocession des forêts affermées (concessions), les indus. triels se voyant offrir à la place des garanties d'approvisionnements pour des périodes de 5 , 10 ans renouvelables. L'autorité gouvernementale assure maintenant l'aménagement fores. tier et la gestion des forêts, tâche qui incombait à l'industrie au temps de l'affermage.

Enfin, on observe une tendance à augmenter la production des forêts privées, autant pour assurer des revenus convenables aux propriétaires généralement agriculteurs que pour réduire les coûts de transport du bois, les forêts privées étant généralement situées à proximité des usines.

On comprendra mieux l'importance du Québec forestier quand on aura examiné le contenu des tableaux 1 et 2 . Le premier montre la répartition par zones d'affectation du territoire du Québec au sud du $52^{\mathrm{e}}$ degré de latitude puisqu'au nord, la forêt clairsemée prend une forme proche de la taïga avant de devenir toundra dans l'extrème nord. 
Tableau $n^{\circ} 1$ Superficies des grandes zones d'affectation du territoire au Québec au 31 janvier 1978 en $\mathrm{km}^{2}$

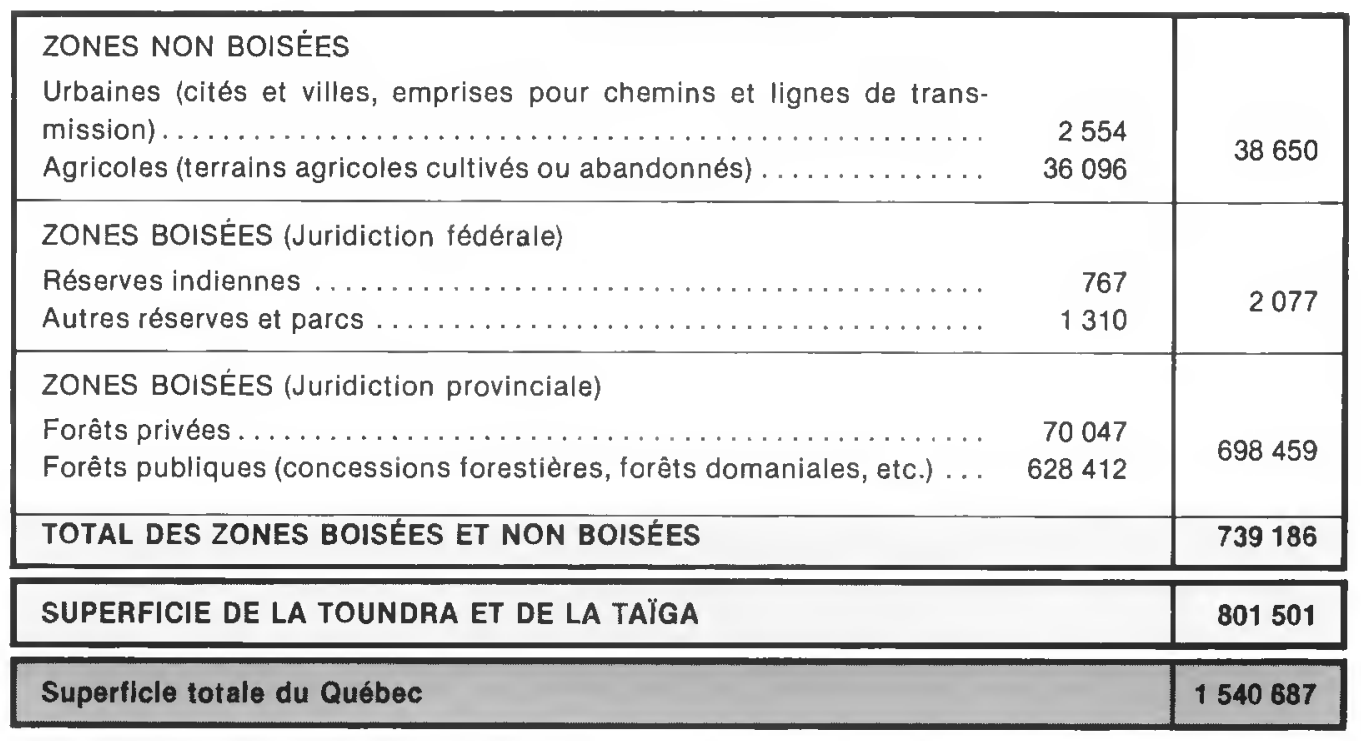

Tableau $n^{\circ} 2$

Superficies et possibilités annuelles de coupes dans les zones boisées de juridiction provinciale (janvier 1978)

\begin{tabular}{|c|c|c|c|c|}
\hline \multirow[t]{2}{*}{$\begin{array}{c}\text { Régimes de propriétés } \\
\text { et de tenure }\end{array}$} & \multicolumn{2}{|c|}{ Superficie en $\mathrm{km}^{2}$} & \multicolumn{2}{|c|}{$\begin{array}{c}\text { Possibilité annuelle de coupe } \\
\text { en } \mathrm{m}^{3}\end{array}$} \\
\hline & Totale & Productivité & Résineux & Feuillus \\
\hline $\begin{array}{l}\text { FORÊTS PRIVÉES ......... } \\
\text { Petites (moins de } 810 \text { ha) } \ldots \\
\text { Grandes (plus de } 810 \text { ha).... }\end{array}$ & $\begin{array}{l}70047 \\
58340 \\
11707\end{array}$ & $\begin{array}{l}60785 \\
50448 \\
10337\end{array}$ & $\begin{array}{l}3561700 \\
2485700 \\
1076000\end{array}$ & $\begin{array}{r}3337100 \\
3136100 \\
201000\end{array}$ \\
\hline $\begin{array}{l}\text { FORÊTS PUBLIQUES ...... } \\
\text { Forêts domaniales ........ } \\
\text { Concessions forestières .... } \\
\text { Forêts cantonales ......... } \\
\text { Forêts expérimentales ..... } \\
\text { Terrains vacants ......... } \\
\text { Lots vacants (colonisation) }\end{array}$ & $\begin{array}{r}628412 \\
337161 \\
204763 \\
4408 \\
329 \\
77954 \\
3797\end{array}$ & $\begin{array}{c}450508 \\
207576 \\
180870 \\
3919 \\
- \\
55351 \\
2792\end{array}$ & $\begin{array}{c}36109000 \\
15220000 \\
17720000 \\
129000 \\
- \\
2856000 \\
184000\end{array}$ & $\begin{array}{c}3991800 \\
1580400 \\
2197200 \\
21100 \\
- \\
114100 \\
79000\end{array}$ \\
\hline TOTAL FORETS ... & 698459 & 511293 & 39670700 & 7328900 \\
\hline
\end{tabular}

